\title{
EDFA Design and Analysis for WDM Optical Systems Based on Modal Multiplexing
}

\author{
Adolfo Fernandes Herbster, \\ Department of Electrical Engineering \\ Federal University of Campina Grande, Campina Grande, PB, Brazil \\ adolfofh@dee.ufcg.edu.br \\ Murilo Araújo Romero \\ Department of Electrical and Computer Engineering \\ University of São Paulo, São Carlos, SP, Brazil \\ murilo.romero@usp.br
}

\begin{abstract}
Modal multiplexing (MDM) is a core technology to allow a capacity increase in modern optical systems. However, in MDM links, the amplified WDM signal suffers not only from lack of spectral gain equalization but also from unequal modal gain. The proper design of the MDM-EDFA is crucial to provide modal gain equalization and to overcome those impairments, thereby ensuring long haul transmission. In this paper, our methodology of project EDFA design for MDM-WDM systems is presented. The technique is based on a figure of merit arising from the rate and propagation equations which describe the EDFA behavior. This figure of merit is then used for the definition of the objective functions employed in the optimization step, which is based on the non-dominated sorting genetic algorithm NSGA-II. Finally, the method is applied to demonstrate gain equalization through the simulation of a WDM optical system composed of two group modes $\left(\mathbf{L P}_{\mathbf{0 1 , s}}\right.$ and $\left.\mathbf{L} \mathbf{P}_{11, \mathrm{~s}}\right)$ with up to 16 channels in each mode. It is demonstrated that, for a MDM-WDM system with optimized EDFAs, the differential spectral gain is the main cause of non-equalization of the amplified signal.
\end{abstract}

Index Terms - Erbium doped fiber amplifier, few-mode fiber amplifiers, genetic algorithm, spatial division multiplexing.

\section{INTRODUCTION}

There has been an increase on the demand of transmission rate in optical networks, due to novel applications, such as high definition video services (e.g. NetFlix) and data center storage (e.g. DropBox and Google Drive). In order to respond to this need, coherent transmission techniques, associated to digital signal processing algorithms, have been developed [1]. As a consequence, the limit of the capacity of the optical channel based on single-mode fiber ( 100 Tbits/s [2]) has already been reached [3-4].

Further progress is possible only if an additional degree of freedom for optical transmission can be exploit. That is why recent research has focused on the development of technologies for coherent optical systems based on Spatial-Division Multiplexing (SDM). In those SDM systems there are two 
basic multiplexing forms (which can also be combined together): (a) through spatially separate cores in a multi-core fiber (MC); and (b) through orthogonal modes in a few-mode fiber (FM). In both cases, for long haul WDM transmission, optical amplification remains an essential part of the optical system.

Specifically, in the particular case of few-mode (FM) systems, for an efficient WDM amplification, the EDFA must present high modal gain and low differential modal gain (DMG), as well as the usual requirement of equalized spectral gain (DSG) across the C-band. Because of its complexity, the design of FM-EDFAs than single-mode amplifiers and the design of FM-EDFAs for WDM signals has recently received major attention [5-6].

The first methodology specifically focused on the FM-EDFA design for WDM systems is described in [7]. An annular doping profile with uniform fiber refractive index profile (FRIP) is proposed in this paper, allowing satisfactory gain equalization between the group modes $\mathrm{LP}_{01, \mathrm{~s}}$ and $\mathrm{LP}_{11, \mathrm{~s}}$ for six WDM channels, namely a DMG value of $\sim 2 \mathrm{~dB}$ associated to gains values higher than $20 \mathrm{~dB}$. The pump signal was composed only by the $\mathrm{LP}_{01, \mathrm{p}}$ mode.

Alternatively, Kang et al proposed an annular doping profile with uniform FRIP and non-centered cladding pump for the equalization of 15 channels multiplexed in four group modes $\left(\mathrm{LP}_{01, \mathrm{~s}}, \mathrm{LP}_{11, \mathrm{~s}}\right.$, $\mathrm{LP}_{21, \mathrm{~s}}$ and $\mathrm{LP}_{02, \mathrm{~s}}$ ) [8]. An optimization procedure concerning the doping profile, based on genetic algorithms, is adopted. The average mode gain is higher than $20 \mathrm{~dB}$, with DMG lower than $1 \mathrm{~dB}$. However, the pump signal intensity is too high $(2.5 \mathrm{~W})$.

Next, in [9], the amplifier is designed to amplify a WDM signal with four channels in four modes. The maximum gain difference between two distinct wavelengths is lower than $3.2 \mathrm{~dB}$ with an average gain of about $21 \mathrm{~dB}$. The geometry of the doped fiber is defined by 19 microstructure cells and the doping profile is optimized through the gradient descent optimization algorithm (GDOA).

Although it presents good results, the optimization method used in [8] and [9] require a fairly timeconsuming numerical solution for the rate and propagation equations describing the behavior of the FM-EDFA. For WDM signals, the optimization procedure significant computational effort, since the necessary processing is much higher than that in the single channel case. As a consequence, it is usual that the investigations reported in the literature focus on a limited search space and/or rely on the predefinition of the pump mode, specified at the beginning of the algorithm.

A new methodology for an FM-EDFA project for WDM signal is proposed in this paper. The approach is based on a technique presented by us in reference [15], originally for the design of singlechannel FM-EDFAs. Specifically, in our methodology, the rate and propagation equations are used only as a starting point, to derive a simplified figure of merit, which relates the output characteristics of the FM-EDF to the set of modes composing the input and pump signals. By using this figure of merit, computational effort is greatly reduced, allowing a generalized optimization process to be carried out by means of the non-dominated sorting genetic algorithm II (NSGA-II) [10].

Here, this methodology is applied to extend the analysis to the differential modal and spectral gain 
of a signal composed of up to 16 channels per mode. The outline of the paper is given as follows. The parameters which are used to evaluate the amplifier performance, such as DMG and DSG, are defined in section II. Next, the figure of merit for WDM signals is determined in section III. This parameter is used in the optimization stage, described in the same section. The FM-EDFA design for WDM signals is carried out in section IV. The performance of the designed amplifier is analyzed in the stand-alone form, as well as inserted into the WDM system.

\section{PERFORMANCE PARAMETERS}

The four basic parameters for the evaluation of an FM-EDFA performance are gain, noise figure, gain efficiency and differential spectral gain (DSG). In conventional WDM systems, the gain difference between two channels is calculated as

$$
\operatorname{DSG}(m)=\max _{\lambda_{i} \neq \lambda_{j}}\left|\mathrm{G}_{d B}\left(\lambda_{i}\right)-\mathrm{G}_{d B}\left(\lambda_{j}\right)\right|,
$$

where $\mathrm{G}_{d B}\left(\lambda_{i}\right)$ represents the gain (in $\mathrm{dB}$ ) in the $\lambda_{i}$ wavelength.

For the FM-EDFA, three new performance parameters are required, namely, the differential modal gain $(\mathrm{DMG})$, gain excursion $(\Delta \mathrm{G})$ and differential modal noise figure (DMNF). In this paper, the parameter related to the noise figure will not be addressed. On the other hand, DMG is defined as the differential gain between two points of the spectrum of the amplified optical modes, as [11]

$$
\operatorname{DMG}(\lambda)=\max _{m \neq n}\left|\mathrm{G}_{d B}(m, \lambda)-\mathrm{G}_{d B}(n, \lambda)\right|,
$$

where $m$ and $n$ represent two allowed signal modes of the FM-EDFA.

For long range systems, the differential gain (DSG and DMG) must be kept the minimum possible. Fig. 1 illustrates the typical optical gain of a FM-EDFA, taking into account an optical input signal composed of four modes $\left(\mathrm{LP}_{01}, \mathrm{LP}_{02}, \mathrm{LP}_{11}\right.$ and $\left.\mathrm{LP}_{21}\right)$ and 32 equally spaced channels between 1530 and $1561 \mathrm{~nm}$. Ideally, both the DSG and the DMG parameters must be zero along the optical band of interest. However, in the example, the value of DSG reaches $7.8 \mathrm{~dB}$ (for $\mathrm{LP}_{01}$ mode) while the DMG can be as high as $11.02 \mathrm{~dB}$ (at $1531 \mathrm{~nm})$.

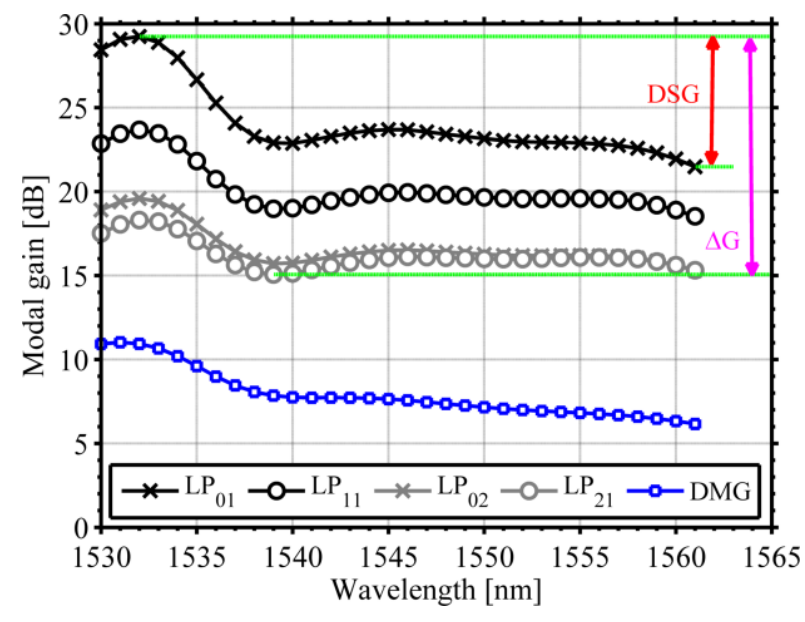

Fig. 1: Performance parameters of the FM-EDFA. 
Finally, the $\Delta \mathrm{G}$ parameter represents the maximum differential gain between two different wavelengths, regardless of the spatial mode, and mathematically defined as [11]:

$$
\Delta \mathrm{G}=\max _{\substack{m \neq n \\ \lambda_{i} \neq \lambda_{j}}}\left|\mathrm{G}_{d B}\left(m, \lambda_{i}\right)-\mathrm{G}_{d B}\left(n, \lambda_{j}\right)\right| .
$$

Accordingly to Fig. 1, the channel $1539 \mathrm{~nm}$ of the mode $\mathrm{LP}_{21}(15.06 \mathrm{~dB})$ exhibits the minimum gain, while the maximum gain is obtained by channel $1532 \mathrm{~nm}$ of the $\mathrm{LP}_{01}$ mode $(29.04 \mathrm{~dB})$. Therefore, $\Delta \mathrm{G}$ is about $14 \mathrm{~dB}$. It is important to note that both the DMG and the DSG parameters contribute to raise the value of $\Delta \mathrm{G}$.

\section{OPTIMIZATION FORMALISM AND METHODOLOGY}

In this paper, the FM-EDFA is considered a two-level system. The FM-EDF is also taken as weakly guided and the propagating modes are described as linearly polarized (LP) [12]. The fiber is considered perfectly symmetrical and coupling effects between spatial modes are disregarded. Optical losses can be also neglected due to the small length of the doped fiber and the pump signal is considered as monochromatic. Our approach is to provide a simplified figure of merit to allow an exhaustive and comprehensive search on the all possible doping profile geometries. With this goal in mind, the figure of merit is derived accounting for the generation of the amplified spontaneous emission (ASE) [13], which is disregarded in a preliminary evaluation of the FM-EDFA performance. However, after the figure of merit is used to select the most promising doping profiles, further analysis and final performance assessment do include the ASE effects.

The derivation of our figure of merit starts by the rate equations, defined in [14] for the two-level system. As a result of above simplifications, those equations are rewritten as:

$$
\begin{gathered}
\frac{N_{1}(\rho, \phi, z)}{N_{0}(\rho, \phi)}=\frac{\frac{h}{\tau}+\sum_{i=1}^{N_{s}} \frac{\sigma_{s, i}^{e}}{v_{s, i}} P_{s, i} \Gamma_{s, i}}{\frac{h}{\tau}+\sum_{i=1}^{N_{s}} \frac{\left(\sigma_{s, i}^{e}+\sigma_{s, i}^{a}\right)}{v_{s, i}} P_{s, i} \Gamma_{s, i}+\frac{\sigma_{q}^{a}}{v_{p}} P_{p} \Gamma_{p}}, \\
\frac{N_{2}(\rho, \phi, z)}{N_{0}(\rho, \phi)}=\frac{\sum_{i=1}^{N_{s}} \frac{\sigma_{s, i}^{e}}{v_{s, i}} P_{s, i} \Gamma_{s, i}+\frac{\sigma_{q}^{a}}{v_{p}} P_{p} \Gamma_{p}}{\frac{h}{\tau}+\sum_{i=1}^{N_{s}} \frac{\left(\sigma_{s, i}^{e}+\sigma_{s, i}^{a}\right)}{v_{s, i}} P_{s, i} \Gamma_{s, i}+\frac{\sigma_{q}^{a}}{v_{p}} P_{p} \Gamma_{p}} .
\end{gathered}
$$

The variables $N_{1}(\rho, \phi, z)\left[\mathrm{m}^{3}\right]$ and $N_{2}(\rho, \phi, z)\left[\mathrm{m}^{3}\right]$ represent the ion density in the fundamental and metastable levels, respectively, while $N_{0}(\rho, \phi)\left[\mathrm{m}^{3}\right]$ is the distribution of the ion density of the FMEDF (doping profile). Subindex $p$ and $s$ represent variables related to pump and input signals, respectively. The absorption and emission cross section are defined by $\sigma^{a}\left[\mathrm{~m}^{2}\right]$ and $\sigma^{e}\left[\mathrm{~m}^{2}\right]$, respectively, $\tau$ [s] represents the lifetime of the erbium ions of the metastable state, $h$ [J.s] represents Planck's constant and $v_{t}$ represents the frequency of the optical component $t$. The variable $P_{t}(z)[\mathrm{W}]$ represents the signal intensity $t$ at position $z[\mathrm{~m}]$ of FM-EDF and variable $\Gamma_{t}(\rho, \phi)\left[\mathrm{m}^{2}\right]$ represents the 
normalized modal-intensity distribution. The maximum number of components $i$ of the input signal is defined by $N_{s}$.

In order to complete the model for the FM-EDFA, while simultaneously taking into account the simplifications proposed in this section, the input signal propagating equations defined in [14] are rewritten as

$$
\frac{\mathrm{d} P_{s, i}}{\mathrm{~d} z}=\underbrace{-P_{s, i} \frac{h \sigma_{s, i}^{a}}{\tau} \int_{0}^{2 \pi a} \int_{0}^{a} \frac{N_{0}(\rho, \phi) \Gamma_{s, i}(\rho, \phi)}{\varphi(\rho, \phi, z)} \rho \mathrm{d} \rho \mathrm{d} \phi}_{\eta_{1}}+\underbrace{P_{s, i} P_{p} \frac{\sigma_{s, i}^{e} \sigma_{p}^{a}}{v_{p}} \int_{0}^{2 \pi a} \int_{0}^{a} \frac{N_{0}(\rho, \phi) \Gamma_{s, i}(\rho, \phi) \Gamma_{p}(\rho, \phi)}{\varphi(\rho, \phi, z)} \rho \mathrm{d} \rho \mathrm{d} \phi}_{\eta_{2}},
$$

where

$$
\varphi(\rho, \phi, z)=\frac{h}{\tau}+\sum_{x=1}^{N_{s}} \frac{\left(\sigma_{s, x}^{a}+\sigma_{s, x}^{e}\right)}{v_{s, x}} P_{s, x} \Gamma_{s, x}+\frac{\sigma_{p}^{a}}{v_{p}} P_{p} \Gamma_{p} .
$$

The first portion at the RHS of Eq. 6, called $\eta_{1}$, contributes to the power decrease of $\mathrm{i}^{\text {th }}$ input signal, due to the absorption of the optical signal. Meanwhile, the second portion at the RHS of Eq. 6, called $\eta_{2}$, contributes to the power of $\mathrm{i}^{\text {th }}$ input signal. The increase of the optical power is proportional to both the intensities of the input $\left(P_{s, i}\right)$ and pump $\left(P_{p}\right)$ signals, as well as to the integrals themselves. When the signal can be considered as a single wavelength channel and the optical amplifier operates in the unsaturated regime, component $\eta_{1}$ is neglected and the simplified equation describing the propagation of the signal is simply defined by $\eta_{2}$.

In this condition, a useful figure of merit concerning the amplifier gain was previously defined by us as [15]

$$
\eta_{s, i}^{p}=\int_{0}^{2 \pi} \int_{0}^{a} N_{0}(\rho, \phi) \Gamma_{s, i}(\rho, \phi) \Gamma_{p}(\rho, \phi) \rho \mathrm{d} \rho \mathrm{d} \phi
$$

\section{A. Figure of merit for WDM signals}

In the case of WDM signals, the set of propagation equations, defined by Eqs. 6 and 7, also includes the added term:

$$
\eta_{3}=\sum_{x=1}^{N_{s}}\left[P_{s, x} P_{s, i} \frac{\left(\sigma_{s, x}^{a} \sigma_{s, i}^{e}-\sigma_{s, x}^{e} \sigma_{s, i}^{a}\right)}{v_{s, x}} \int_{0}^{2 \pi} \int_{0}^{a} \frac{N_{0}(\rho, \phi) \Gamma_{s, i}(\rho, \phi) \Gamma_{p}(\rho, \phi)}{\varphi(\rho, \phi, z)} \rho \mathrm{d} \rho \mathrm{d} \phi\right] .
$$

This new term added to Eq. 6 corresponds to the process of stimulated emission, rising from the absorption of the input signal. This component is zero when the wavelength of component $i$ is close to the wavelength of component $x\left(\sigma_{s, x}^{a} \sigma_{s, i}^{e}-\sigma_{s, x}^{e} \sigma_{s, i}^{a}=0\right)$.

The value of $\eta_{3}$ is proportional to the product of optical intensities for the components of the input signal $\left(P_{s, x} P_{s, i}\right)$. Therefore, at the fiber input, the magnitude of $\eta_{3}$ is negligible when compared to $\eta_{2}$. However, in the saturation regime of the FM-EDF, when the amplitude of input signal amplitude is high, component $\eta_{3}$ must be taken into account. On the other hand, similarly to the single channel case, $\eta_{1}$ and $\eta_{3}$ can be disregarded when the FM-EDFA operates in the linear (i.e, unsaturated) region. 
Also, as explained before, the proper design of FM-EDFAs aims at equalized modal gain. In order to assure that the values of modal gains are similar, a sufficient condition is that $d P_{s, i} / d z=d P_{s, i} / d z$, with $i \neq j$. In this case, when considering the optical intensities concerning the signal components of the input signal to be similar, it is necessary that

$$
\sigma_{s, i}^{e} \int_{0}^{2 \pi a} \int_{0}^{a} \frac{N_{0}(\rho, \phi) \Gamma_{s, i}(\rho, \phi) \Gamma_{p}(\rho, \phi)}{\varphi(\rho, \phi, z)} \rho \mathrm{d} \rho \mathrm{d} \phi=\sigma_{s, j}^{e} \int_{0}^{2 \pi} \int_{0}^{a} \frac{N_{0}(\rho, \phi) \Gamma_{s, j}(\rho, \phi) \Gamma_{p}(\rho, \phi)}{\varphi(\rho, \phi, z)} \rho \mathrm{d} \rho \mathrm{d} \phi .
$$

The previous expression can be explored by highlighting three particular cases. The first case consists of an input signal with only one spatial mode, such as the $\mathrm{LP}_{01, \mathrm{~s}}$ mode, and different channels, for example, 1530 and $1550 \mathrm{~nm}$. In this case, the integral $\eta_{2}$ is nearly identical for all the signal components, because the distribution of normalized intensity for all the channels in the same spatial mode is similar. Accordingly, a sufficient condition for the gain equalization is to assure that the cross sections of the different channels are similar. Unfortunately, complete spectral gain equalization in a given mode is not possible, because it is not possible to make the emission cross section independent from the wavelength, since the value of this parameter depends on the position of energy levels of the erbium ions in the silica matrix [16].

The second case relates to optical channels of same wavelength but distinct spatial modes. Consider, for example, a channel located at $1530 \mathrm{~nm}$ and composed of the spatial modes $\mathrm{LP}_{01, \mathrm{~s}}$ and $\mathrm{LP}_{11, \mathrm{~s}}$. For this case, the emission cross sections are similar $\left(\sigma_{s, i}^{e}=\sigma_{s, j}^{e}\right)$ and Eq. 10 must be satisfied for the design of the FM-EDF, by careful selection of the Erbium doping profile as well as the choice of the pump signal mode.

Finally, the third case is a generalization of the previous two, that is, the input signal is composed of different optical channels and of different spatial modes, for example, channels at 1530 and $1550 \mathrm{~nm}$ for spatial modes $\mathrm{LP}_{01, \mathrm{~s}}$ and $\mathrm{LP}_{11, \mathrm{~s}}$. In this case, besides the similarity between the overlap integrals, it is necessary that $\sigma_{s, i}^{e}=\sigma_{s, j}^{e}$ (for different signal wavelength components).

Concerning this required spectral equalization, in the unsaturated region, the amplifier shows maximum gain close to $1530 \mathrm{~nm}$ and a relatively flat region between 1540 and $1560 \mathrm{~nm}$. However, in moderate inversion condition, the region close to $1530 \mathrm{~nm}$ presents higher attenuation, due to the higher value of the absorption cross section [16]. Therefore, controlling the equalization of the amplifier simply through the inversion control is not effective for long distance systems. This is because the link reach and, consequently, the link loss are a paramount factor to determine the level of inversion of the fiber. In other words, non-intentional variations of the loss of links generate nonequalized gain. To overcome this difficulties a multi-objective optimization procedure for the design of WDM FM-EDFAs is provided below. 


\section{B. Multi-objective optimization}

The methodology applied to the FM-EDFAs design for WDM signals is presented in this section. The methodology can be used in single channel as well as WDM systems, being divided into four phases: (a) parameters definition, (b) solution of the multi-objective problem, (c) determination of the FM-EDF optimum length, and (d) analysis of the amplifier performance.

1. Parameters definition: parameters concerning the input signal, the FM-EDF and the pump signal are defined in the phase. The modes are characterized by the distribution of normalized intensity, optical power and frequency of each component. Because the wavelength of the pump signal, e.g. $980 \mathrm{~nm}$, is lower than the input signal, e.g. C band, the value of the pump beam normalized frequency is higher, allowing more propagating modes. Desired restrictions on the allowed geometries of the doping profile for the FM-EDF are specified in this phase.

2. Solution of the multi-objective problem: to determine the pumping mode and the variables that specify the geometry of the FM-EDF profile, in order to reduce the differential modal gain (DMG) without lowering the absolute gain, is translated into a multi-objective optimization problem (MOOP). The objective functions, which describe the design problem as a trade-off between the conflicting goals (high modal gain and low DMG), are defined as

$$
\begin{aligned}
& f_{1}(\mathbf{x})=-\bar{\eta}, \\
& f_{2}(\mathbf{x})=\sigma_{\eta},
\end{aligned}
$$

where $\bar{\eta}$ and $\sigma_{\eta}$ are the average and standard deviation of our figure of merit, defined by Eq. 8. The definition of the MOOP in terms of the figure of merit defined by Eq. 8 causes the reduction of processing time required for the FM-EDFA design, allowing the algorithm NSGA-II [10] to be employed for MOOP solution. Although there is no guarantee of obtaining the global optimum, optimum local solutions are easily found.

3. Determination of the FM-EDF optimum length: the third phase determines the FM-EDF ideal length. In a WDM input signal, as the inversion of the Er ions population affects the value of the differential spectral gain (DSG), for each level of intensity of the input and pump signals, there is an optimum length. The length in which the FM-EDFA displays lower DSG and DMG and higher average gain is chosen as the ideal length.

4. Performance analysis of the FM-EDFA: in the final phase, the main parameters of the FMEDFA (average gain, DMG, DSG and $\Delta \mathrm{G}$ ) are determined for a set of values of intensity of the input signal and pump power by the rigorous solution of propagation and rate equations. In case the design target goals are not achieved, the pump mode and profile determined by the algorithm NSGA-II are disregarded. New simulations are performed up to the point where a solution meeting all design objectives is achieved. 


\section{Simulation AND ANALYSIS}

In this section, the $\mathrm{LP}_{l m, \mathrm{p}}$ and $\mathrm{LP}_{l m, \mathrm{~s}}$ notations are used to denote the spatial mode $\mathrm{LP}_{l m}$ in the wavelength of the pump signal, e.g. $980 \mathrm{~nm}$, and the C-band input signal, respectively. Likewise, the $\mathrm{LP}_{l m a}$ and $\mathrm{LP}_{l m b}$ notations represent the degenerated $\mathrm{LP}_{l m}$ mode of odd and even orientations, respectively.

The pump signal is considered monochromatic and co-propagating, with wavelength equal to 980 $\mathrm{nm}$. Taking into account the degenerated modes, the pump signal is represented by

$$
\mathrm{LP}_{l m, \mathrm{p}}=\frac{1}{2}\left(\mathrm{LP}_{l m a, \mathrm{p}}+\mathrm{LP}_{l m b, \mathrm{p}}\right) .
$$

The pump signal is modal and polarization multiplexed. For the non-degenerated modes, the signal is represented by

$$
\mathrm{LP}_{0 m, \mathrm{~s}}=\left(\mathrm{LP}_{0 m x, \mathrm{~s}}+\mathrm{LP}_{0 m y, \mathrm{~s}}\right),
$$

while the degenerated modes are defined by

$$
\mathrm{LP}_{l m, \mathrm{~s}}=\frac{1}{2}\left(\mathrm{LP}_{l m a x, \mathrm{~s}}+\mathrm{LP}_{l m a y, \mathrm{~s}}+\mathrm{LP}_{l m b x, \mathrm{~s}}+\mathrm{LP}_{l m b y, \mathrm{~s}}\right),
$$

in which $\mathrm{LP}_{\text {lmax }, \mathrm{s}}$ and $\mathrm{LP}_{\text {lmay,s }}$ represent polarization $x$ and $y$ of the degenerated mode $\mathrm{LP}_{\text {lma,s }}$ respectively.

In the sections to follow, first, the FM-EDFA is designed considering a WDM signal composed by two group modes $\left(\mathrm{LP}_{01, \mathrm{~s}}\right.$ and $\left.\mathrm{LP}_{11, \mathrm{~s}}\right)$. Next, an optical system is simulated in order to analyze the performance of the amplified signal, considering its average gain, differential modal and spectral gain and gain excursion.

\section{A. FM-EDFA for WDM signals}

In this subsubsection, each mode of the input signal is composed by 16 spaced channels between 1530 and $1550 \mathrm{~nm}$. ASE noise is considered in the simulations. The core radius and numerical aperture (NA) of the FM-EDF used in the simulations are equal to $7.18 \mu \mathrm{m}$ and 0.13 respectively. The refractive index difference at the core-cladding interface and the erbium ion density are $5.91 \times 10^{-3}$ and $5 \times 10^{24}\left[\mathrm{~m}^{-3}\right]$. The target geometry of the FM-EDF doping profile is pre-selected as annular, or, in other words, defined by two concentric rings of radius $\mathrm{x}_{1}$ and $\mathrm{x}_{2}$. The values of the emission and absorption cross sections are extracted from [17] (corresponding to the R37003X fiber).

The pump signal wavelength is $980 \mathrm{~nm}$, and the normalized frequency is 5.98 , in such way that there are six spatial propagation modes $\left(\mathrm{LP}_{01, \mathrm{p}}, \mathrm{LP}_{11, \mathrm{p}}, \mathrm{LP}_{21, \mathrm{p}}, \mathrm{LP}_{02, \mathrm{p}}, \mathrm{LP}_{31, \mathrm{p}}\right.$ and $\left.\mathrm{LP}_{12, \mathrm{p}}\right)$. The methodology described in the previous section was then applied and the resulting radii that define the Er doping profile are $\mathrm{x}_{1}=1.91 \mu \mathrm{m}$ and $\mathrm{x}_{2}=7.18 \mu \mathrm{m}$. The optimization algorithm NSGA-II also identifies the $\mathrm{LP}_{11, \mathrm{p}}$ mode as the most suitable pump mode to promote the amplifier performance.

The values of average gain and DMG as a function of the length of the FM-EDFA are illustrated in Fig. 2(a). The pumping intensity is $275 \mathrm{~mW}$ and the power per channel is $-22 \mathrm{dBm}$ (total of -10 $\mathrm{dBm} /$ mode). Each point in the graphs of Fig. 2(a) represents the average and the standard deviation of 
our figure of merit, concerning the amplified signal composed by 16 channels.

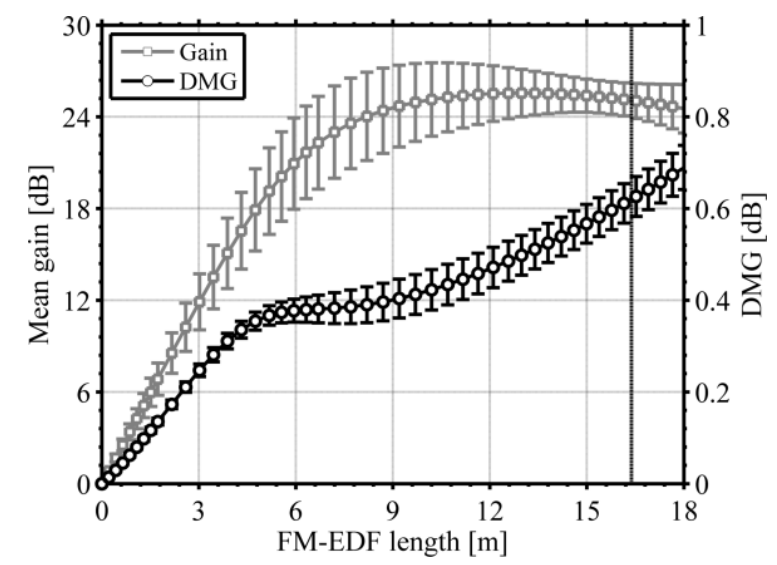

(a)

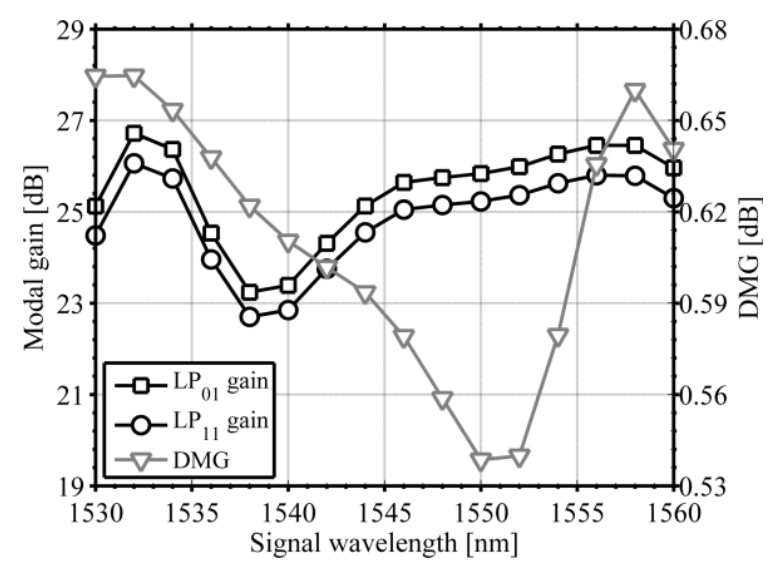

(b)

Fig. 2: Performance of the FM-EDFA: (a) average gain and DMG as a function of the length of the FM-EDF and (b) modal gain and DMG as a function of the wavelength with ideal FM-EDF length equal to $16.4 \mathrm{~m}$. The power of the input signal per channel is $-22 \mathrm{dBm}$ and the pump power is $275 \mathrm{~mW}$.

The average gain, whose maximum value is $25.5 \mathrm{~dB}$, is directly proportional to the length of the FM-EDF up to 12.8 meters. After this point, the value of the average gain decreases, due to the absorption of the amplified optical signal. However, the minimum value of the standard deviation for our figure of merit occurs at the length of 16.4 meters. As a consequence, this length corresponds to a minimum value of DSG, equal to $3.3 \mathrm{~dB}$, while the corresponding average gain is $25 \mathrm{~dB}$, only $2 \%$ lower than the maximum average gain $(25.5 \mathrm{~dB})$. Although the increase of the DMG is lower than the $0.15 \mathrm{~dB}(0.57 \mathrm{~dB}$ and $0.68 \mathrm{~dB}$ in 12.8 meters and 16.4 meters, respectively) for maximum gain operation, the variation of the DSG is quite high, of $1.9 \mathrm{~dB}(5.3 \mathrm{~dB}$ and $3.4 \mathrm{~dB}$ in $12.8 \mathrm{~m}$ and $16.4 \mathrm{~m}$ respectively), justifying the choice of the FM-EDF ideal lengths $16.4 \mathrm{~m}$. In what follows, this initial amplifier design is called design $A$.

The modal gain spectrum and the value of the DMG are shown in Fig. 2(b), taking the same optical power of the input and pump signals used when considering the FM-EDF length equal to 16.4 meters. The difference in modal gain is lower than $0.68 \mathrm{~dB}$ along the whole C-band, therefore being lower than the DSG. Then, in this case, the main factor of optical signal degradation is the high value of the differential spectral gain, penalizing the channels located in the spectral region around $1540 \mathrm{~nm}$, because the gain level is about $2 \mathrm{~dB}$ lower than observed in the spectral range between channels 1545 and $1560 \mathrm{~nm}$.

The ideal length of the FM-EDF varies according the power of the input and pump signals, due to the change on the inversion level. Fig. 3 shows the performance of the design A FM-EDFA as function of the pump power and of the power of the input signal. In the first case (Fig. 3(a)), the pump intensity of the $\mathrm{LP}_{11, \mathrm{p}}$ mode varies between 100 and $345 \mathrm{~mW}$ and the power per channel equals to -22 $\mathrm{dBm}$. The higher the pump intensity the higher the fiber length necessary to absorb the pump signal as well as to guarantee a moderate inversion along the fiber. Likewise, the average gain and the DSG are proportional to the pump intensity. However, the value of DMG is inversely proportional to the 
optical pumping level.

The performance of the FM-EDF as a function of the input signal power is shown in Fig. 3(b), taking into account the pump intensity $275 \mathrm{~mW}$. Similarly to what is observed in Fig. 3(a), the ideal length varies inversely to the input signal level.

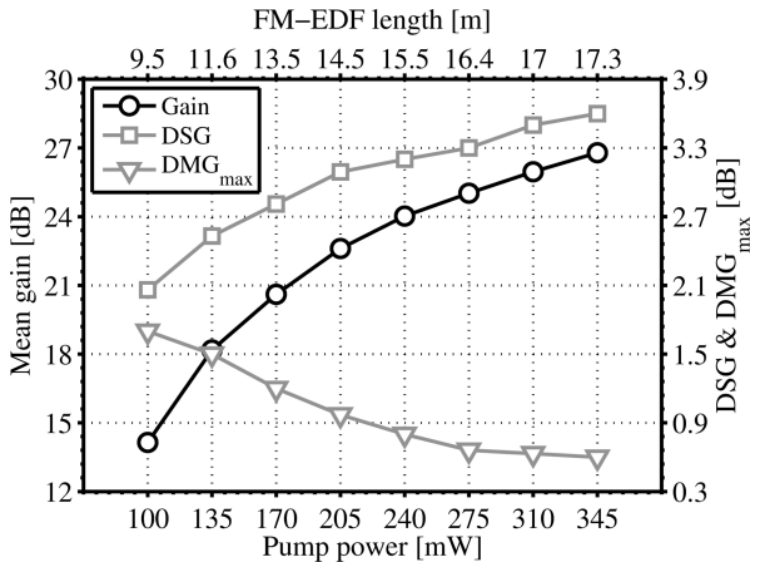

(a)

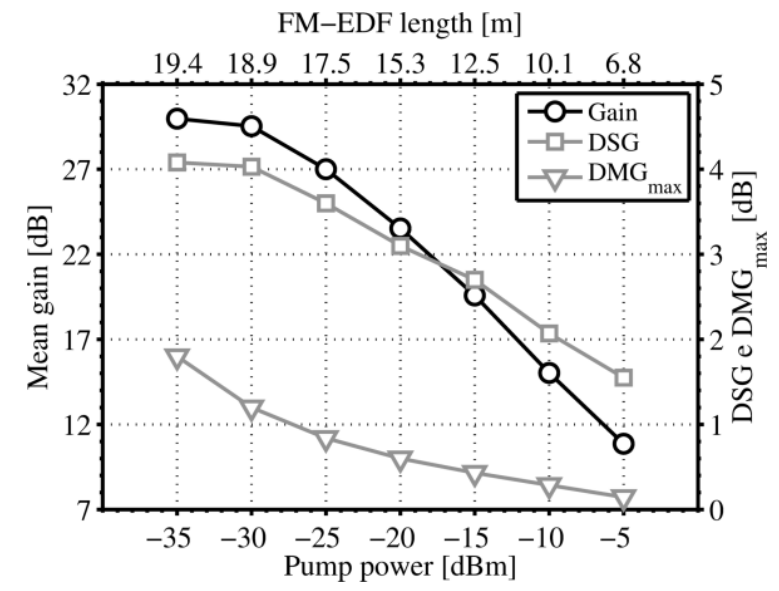

(b)

Fig. 3: Performance of the FM-EDFA as function (a) of the pump power of the $\mathrm{LP}_{11 \mathrm{p}}$ mode and $-22 \mathrm{dBm}$ per channel and (b) power of the input signal per channel and $275 \mathrm{~mW}$ of pump intensity.

\section{B. Optical MDM-WDM systems}

The block diagram of the simulated system is shown in Fig. 4. Since the optical fiber is not explicitly included in the diagram, only attenuation effects are considered in the analysis. In the simulation, three WDM transmitters, one for each mode $\left(\mathrm{LP}_{01}, \mathrm{LP}_{11 \mathrm{a}}, \mathrm{LP}_{11 \mathrm{~b}}\right.$, respectively) generate the optical signal with 16 equally spaced channels between 1530 and $1560 \mathrm{~nm}$. The optical power per channel equals to $-15 \mathrm{dBm}$, representing $-3 \mathrm{dBm}$ per mode. The WDM optical signals are multiplexed through a lossless modal multiplexer (MMUX) and the FM-EDFA placed after the MMUX ensures that the optical power at the link input is $5 \mathrm{dBm} / \mathrm{channel}$.

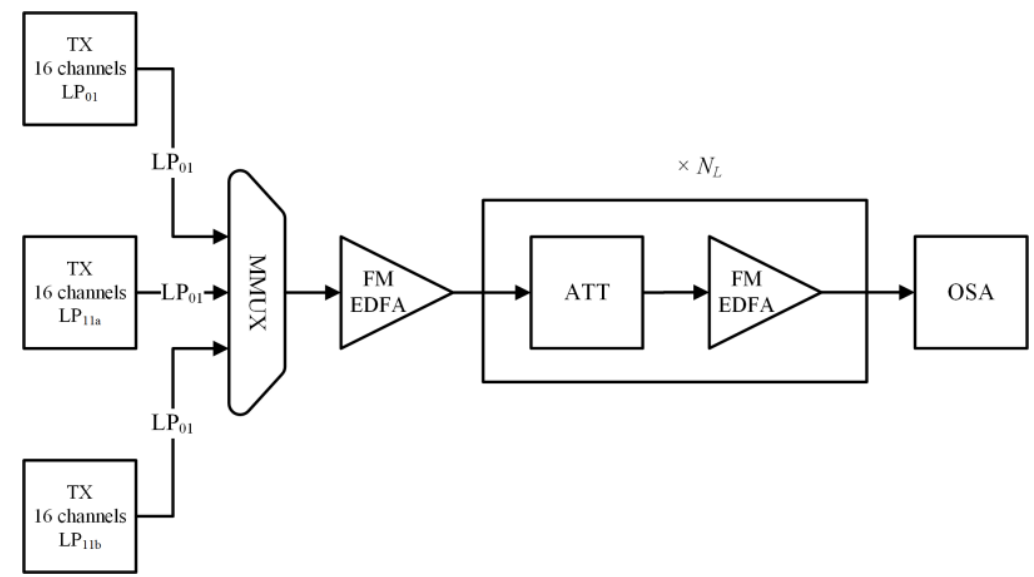

Fig. 4: Block diagram of the simulated communication system, with input signal multiplexed both in the modal and wavelength domains.

The simulated optical link consists of an optical attenuator (ATT), whose attenuation is equal to 20 $\mathrm{dB}$, in series with the design A FM-EDFA described in the previous sub-section ( $20 \mathrm{~dB}$ of gain). This 
set of devices (ATT plus FM-EDFA) is repeated $N_{L}$ times. Finally, an optical spectrum analyzer (OSA) is used to evaluate the properties of the optical signal.

Fig. 5(a) shows the gain spectrum for each mode group of the amplified signal. The analysis focus on two specific points in the system: the outputs of the first and tenth amplifiers, the last case corresponding to the $10^{\text {th }}$ identical span. The maximum values of DSG and of DMG of the amplified optical signal are $2.8 \mathrm{~dB}$ and $0.43 \mathrm{~dB}$, respectively, in the first passing through the amplifier. After ten rounds of amplification, these values increase to $26 \mathrm{~dB}$ and $3.7 \mathrm{~dB}$.

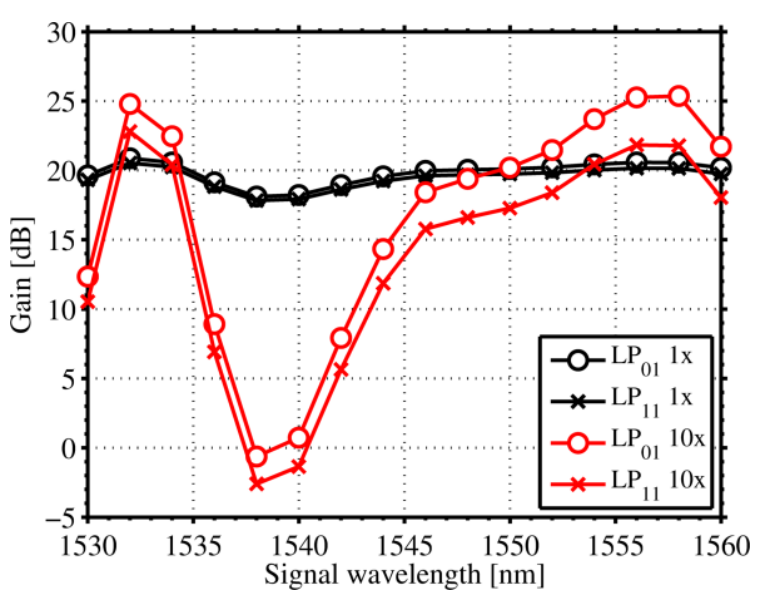

(a) 16 channels in 1530 and $1560 \mathrm{~nm}$

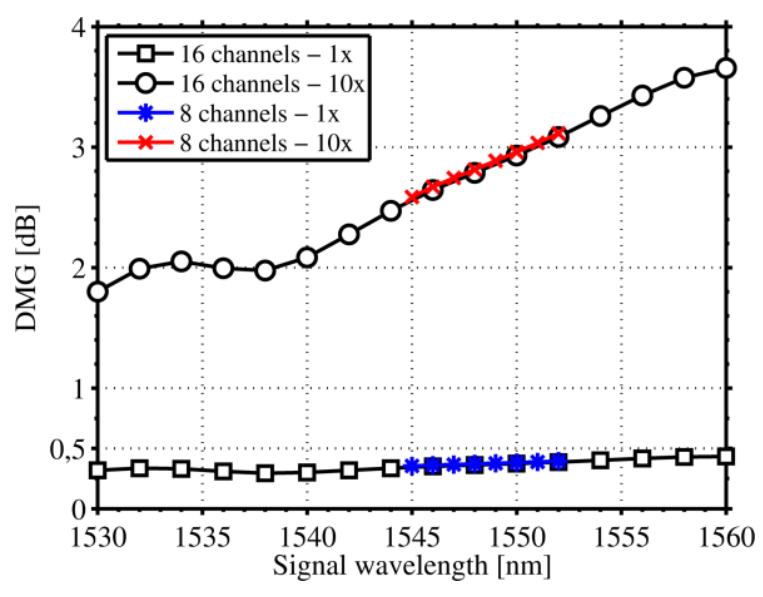

(c)

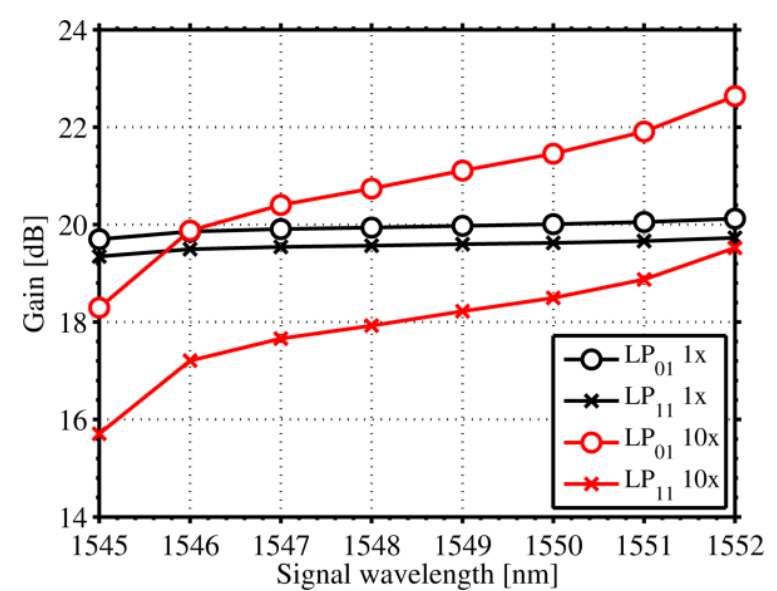

(b) 8 channels in 1545 and $1552 \mathrm{~nm}$

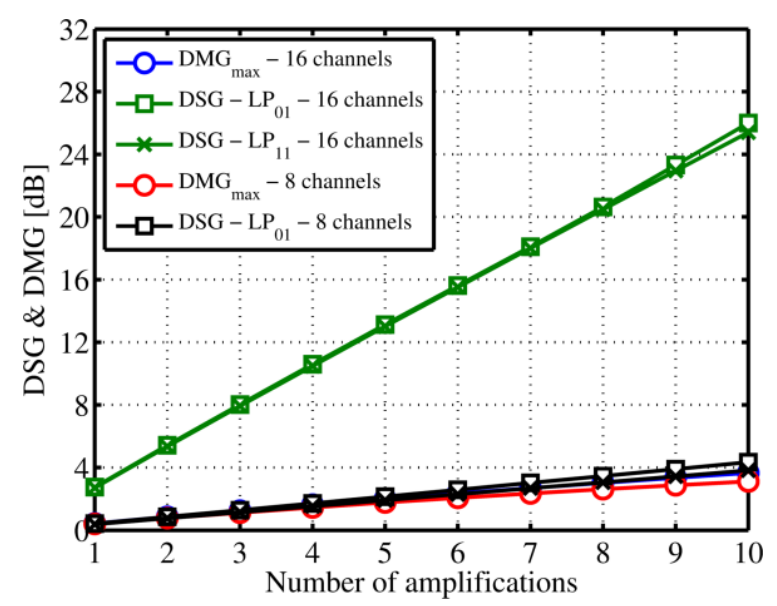

(d)

Fig. 5: Performance of the simulated optical system, in which the parameters are extracted from the output signals of the first (1x) and of the tenth (10x) amplifier (design A) of the optical link, considering two input signals, composed by 16 or 8 channels.

The results indicate that the values of DSG can become prohibitively high across the overall Cband. In order to minimize the effects of the DSG on the amplified signal, a possible solution is to restrict signal transmission to the spectral region between 1545 and 1552 to take advantage of its higher level of gain equalization. In this case, each optical signal generated was composed of eight equally spaced channels, with optical power of $-12 \mathrm{dBm}$ per channel, representing $-3 \mathrm{dBm}$ of optical intensity per mode.

The resulting gain spectra of the amplified signal modes are illustrated in Fig. 5(b). The maximum 
values of DSG and of DMG of the amplified optical signal in the first passing are $0.42 \mathrm{~dB}$ and $0.4 \mathrm{~dB}$, respectively. After ten amplifications, these values increase to $4.3 \mathrm{~dB}$ and $3.1 \mathrm{~dB}$. Compared to the previous case, there is a significant decrease on the DSG (4.3 versus $26 \mathrm{~dB}$ ) with little variation on the DMG, albeit at expense of a reduction on the available optical bandwidth.

From the analysis of Fig. 5(c), which shows the DMG of the amplified optical signal as a function of the wavelength, the DMG does not depend on the number of channels (16 or 8 channels) but rather on the power per mode, because the values of the differential modal gain (DMG) are similar in the region between 1545 and $1552 \mathrm{~nm}$.

Next, Fig. 5(d) shows the maximum DSG and DMG as a function of the number of amplification spans. All parameters analyzed are directly proportional to the number of amplification stages and, consequently, to the length of the optical link. The difference between the cases analyzed (16 or 8 channels, respectively) are the values of the DSG, higher for the case in which 16 channels compose the input signal.

The final analysis consists of examining the performance degradation arising from the use of a conventional, uniform doping FM-EDFA, not specifically designed for MDM-WDM optical systems. The resulting lack of gain equalization (expressed in terms of DSG and DMG), as a function of the number of amplifiers along the link, is presented in Fig. 6(a) for an amplified MDM-WDM system considering an FM-EDF of uniform doping profile. The properties of the FM-EDF of uniform profile, such as ions density and numerical aperture, are similar to the fiber used on the design A FM-EDFA of the previous sections. However, in the case discussed here the optimum fiber length is 11.25 meters. This second amplifier design is called design $B$ FM-EDFA.

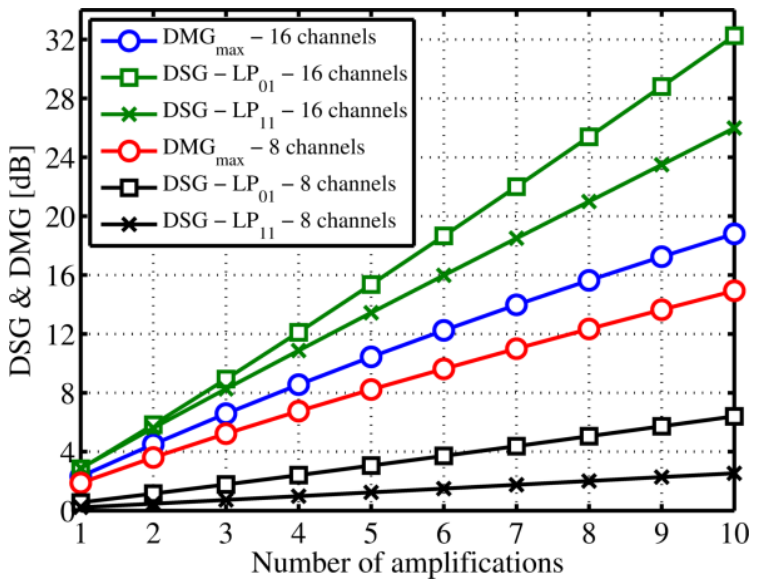

(a)

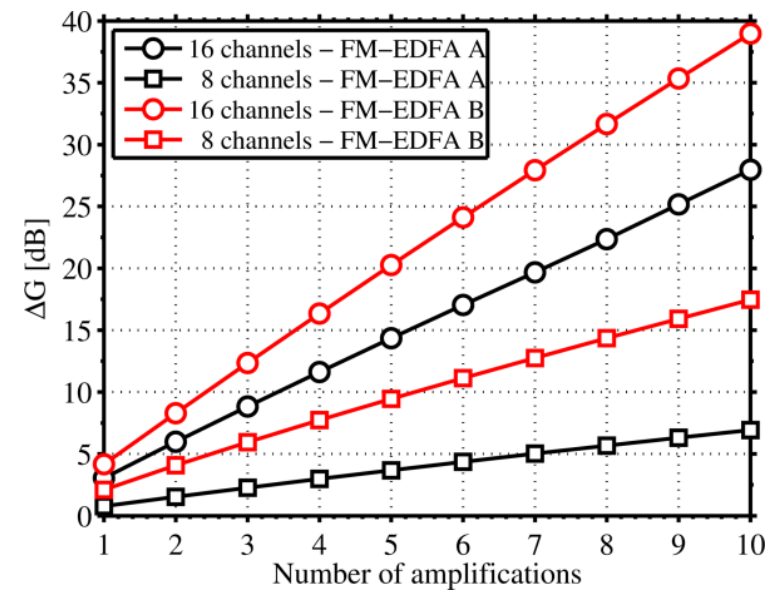

(b)

Fig. 6: (a) DSG and DMG parameters of the amplified optical signal with design B FM-EDFA and (b) maximum variation of gain $\Delta \mathrm{G}$ as a function of the number of amplifiers along the optical link. Two input signals are considered, composed by 16 and 8 WDM channels, respectively, and, in (b), two FM-EDFA are used: model A FM-EDFA A and the uniform doping FMEDFA (model B).

After ten amplifications spans, in the best case scenario ( 8 channels), the maximum values of DSG and DMG are $6.4 \mathrm{~dB}$ and $15 \mathrm{~dB}$, respectively. As expected, since the FM-EDFAs was not designed 
for modal gain equalization, the main source of penalties becomes the DMG (and not the DSG, as in the previous design), clearly demonstrating the need for specific tailoring of the FM-EDFA doping profile in order to assure modal equalization.

The gain excursion, defined in Eq. 3, is illustrated in Fig. 6(b). For this analysis, the amplifiers considered are models A and B, respectively, and the two input optical signals are composed of 8 optical channels with $-12 \mathrm{dBm}$ per channel or 16 optical channels with $-15 \mathrm{dBm}$ per channel. The total power per mode in both cases is $-3 \mathrm{dBm}$. Accordingly to Fig. 5(d), when model A is used and the signal is composed by 16 channels, the raising of $\Delta \mathrm{G}$ can be mostly attributed to the signal DSG. In fact, the value of $\Delta \mathrm{G}$ after ten amplifications is $28 \mathrm{~dB}$, while the maximum values of DSG and DMG are $26 \mathrm{~dB}$ and $3.7 \mathrm{~dB}$ respectively.

In the case where model A FM-EDFA is used and the signal is composed of 8 channels, the contributions to $\Delta \mathrm{G}$ are equally divided between the parameters DSG and DMG, also representing the best system performance. In contrast, accordingly to Fig. 6(a), when model B is used (uniform doping profile) and the input signal composed by 16 channels, the higher portion of $\Delta \mathrm{G}$ still comes from the DSG effect. However, when the signal is composed by 8 channels, the DMG effect provides the major contribution to $\Delta \mathrm{G}$.

\section{CONCLUSION}

The optimum doping profile of FM-EDFAs was investigated by using our proposed figure of merit, a modified overlap integral, to calculate the gain performance of each configuration. The use of the figure of merit greatly reduces computational effort, allowing the designer to employ the NSGA-II genetic algorithm for a complete search of the whole solution space.

It is then possible to propose novel doping profiles, not yet investigated in the literature. An optimum design, considering both pump mode and doping, was provided and a WDM analysis was carried out. When comparing the system performance accordingly to the amplifier model used, it is demonstrated that a specifically designed FM-EDFA is essential for an improved performance of the MDM-WDM optical system since FM-EDFAs with uniform doping profiles cannot provide adequate levels of gain equalization.

\section{ACKNOWLEDGMENT}

This work was partially supported by CNPq (Brazilian Research Council).

\section{REFERENCES}

[1] S. Savory, "Digital coherent optical receivers: Algorithms and subsystems," IEEE Journal of Selected Topics in Quantum Electronics, vol. 16, no. 5, pp. 1164-1179, 2010.

[2] R.-J. Essiambre, G. Kramer, P. Winzer, G. Foschini, and B. Goebel, "Capacity limits of optical fiber networks," IEEE Journal of Lightwave Technology, vol. 28, no. 4, pp. 662-701, 2010.

[3] D. Qian, M.-F. Huang, E. Ip, Y.-K. Huang, Y. Shao, J. Hu, and T. Wang, "101.7-Tb/s (370×294-Gb/s) PDM128QAM-OFDM transmission over $3 \times 55-\mathrm{km}$ SSMF using pilot-based phase noise mitigation," in Optical Fiber Communication Conference/National Fiber Optic Engineers Conference 2011. Los Angeles, EUA: Optical Society of America, March 2011, p. PDPB5.

[4] A. Sano, T. Kobayasgi, S. Yamanaka, A. Matsuura, H. Kawakami, Y. Miyamoto, K. Ishihara, and H. Masuda, 
"102.3-Tb/s (224×548-Gb/s) C- and extended L-band all Raman transmission over $240 \mathrm{~km}$ using PDM-64QAM single carrier FDM with digital pilot tone," in Optical Fiber Communication Conference/National Fiber Optic Engineers Conference 2012. Los Angeles, EUA: Optical Society of America, 2012, p. PDP5C.3.

[5] G. Le Cocq, Y. Quiquempois, and L. Bigot, "Optimization algorithm applied to the design of few-mode erbium doped fiber amplifier for modal and spectral gain equalization," IEEE Journal of Lightwave Technology, vol. 33, no. 1, pp. 100-108, 2015.

[6] Q. Kang, E.-L. Lim, F. P. Y. Jung, C. Baskiotis, S. ul Alam, and D. J. Richardson, "Minimizing differential modal gain in cladding-pumped EDFAs supporting four and six mode groups," Optics Express, vol. 22, no. 18, pp. 21 499-21 507, 2014.

[7] Q. Kang, E.-L. Lim, Y. Jung, J. K. Sahu, F. Poletti, C. Baskiotis, S.U. Alam, and D. J. Richardson, “Accurate modal gain control in a multimode erbium doped fiber amplifier incorporating ring doping and a simple LP01 pump configuration," Optics Express, vol. 20, no. 19, pp. 20 835-20 843, September 2012.

[8] Q. Kang, E.-L. Lim, F. P. Y. Jung, C. Baskiotis, S. ul Alam, and D. J. Richardson, "Minimizing differential modal gain in cladding-pumped EDFAs supporting four and six mode groups," Optics Express, vol. 22, no. 18, pp. 21 499-21 507, 2014.

[9] G. L. Cocq, Y. Quiquempois, A. L. Rouge, G. Bouwmans, H. E. Hamzaoui, K. Delplace, M. Bouazaoui, and L. Bigot, "Few mode $\mathrm{Er}^{3+}$-doped fiber with micro-structured core for mode division multiplexing in the C-band," Optics Express, vol. 21, no. 25, pp. 31 646-31 659, Dezembro 2013.

[10] K. Deb, A. Pratap, S. Agarwal, and T. Meyarivan, "A fast and elitist multiobjective genetic algorithm: NSGA-II," IEEE Transactions on Evolutionary Computation, vol. 6, no. 2, pp. 182-197, 2002.

[11] L. Bigot, G. Le Cocq, and Y. Quiquempois, "Few-mode erbium-doped fiber amplifiers: A review," IEEE Journal of Lightwave Technology, vol. 33, no. 3, pp. 588-596, 2015.

[12] D. Gloge, "Weakly guiding fibers," Applied Optics, vol. 10, no. 10, pp. 2252-2258, 1971.

[13] Z. Jiang and J. R. Marciante, "Impact of transverse spatial-hole burning on beam quality in large-mode-area Ybdoped fibers," Journal of the Optical Society of America B, vol. 25, no. 2, pp. 247-254, 2008.

[14] N. Bai, E. Ip, T. Wang, and G. Li, "Multimode fiber amplifier with tunable modal gain using a reconfigurable multimode pump," Optics Express, vol. 19, no. 17, pp. 16 601-16 611, 2011.

[15] A. Herbster and M. A. Romero, "On the design of few-mode Er-doped fiber amplifiers for space-division multiplexing optical communications systems," in Proceedings of SPIE, vol. 9131, 2014, pp. 91 310Y-91 310Y-9.

[16] P. C. Becker, N. A. Olsson, and J. R. Simpson, Erbium-Doped Fiber Amplifiers: Fundamentals and Technology. San Diego, EUA: Academic Press, 1999.

[17] R37003X - OFS, 2014. [Online]. Available: http://www.ofsoptics.com/. 